\title{
Channel Capacity Evaluation of UIC Cables for High Speed Data Transmission Onboard Regional Trains
}

\author{
S. Barmada ${ }^{1}$, M. Tucci ${ }^{1}$ and F. Romano ${ }^{2}$ \\ ${ }^{1}$ DESTEC, University of Pisa, Italy \\ ${ }^{2}$ Trenitalia s.p.a., Italy
}

\begin{abstract}
In this paper the authors perform a detailed analysis relative to the use of the UIC, International Union of Railways, cable as a data transmission channel for information on board regional trains. The analysis is performed evaluating the channel capacity and taking into account the constraints due to the signal integrity preservation of other signals carried by the cable during regular train operation. The analysis is performed modeling the cable as a multiconductor transmission line in the frequency range of interest, evaluating the crosstalk between all the cable's poles.
\end{abstract}

Keywords: data transmission, broadband power line, channel modeling, onboard communication.

\section{Introduction}

Modern railway systems follow the actual trend in the automotive market: the modern traveler expects to be offered services such as onboard entertainment, high speed internet connection, detailed information about the trip etc. In addition, nowadays increase in the need of onboard safety leads to the requirement of onboard cameras located in each hauled stock.

All this new equipment requires one more dedicated cable networks but is characterized by the following drawback: new cables mean additional costs, weight and space (the latter ones being secondary problems in railways systems if compared to cars, airplanes or satellites). This holds for new trains, while for old trains (for instance regional trains) the revamping procedure could be extremely expensive, basically making it impossible to be realized.

The use of Broadband Power Line (BPL, or high speed Power Line Communications, PLC) in vehicles is an application that has been investigated by many reasearchers lately (see for instance [1] - [7]), showing that this technology 
could be of great help in reducing the cable harness. The authors have already performed a feasibility study and selected a potential candidate cable for carrying the broadband signal, mainly the UIC cable constituting the remote control and communication line ([8] - [10]).

The rationale behind this choice is the following:

- This electrical connection is extremely common in hauled stock and it is unified by regulation [8].

- It connects the whole length of the train and, for safety and functional reasons, particular care is taken in order to avoid any possible disconnection.

- Its use could lead to an easy procedure for revamping older trains a reduced cost.

In [9] and [10] the cable has been tested and its electrical parameters have been measured and simulated, in addition some simulations relative to a data transmission are shown.

In this work the authors evaluate the channel capacity (hence the maximum data rate available) as a function of the noise produced by the other signals carried by the cable. The above described cable is a multiconductor transmission line and the crosstalk phenomena can create undesired voltages and currents which might disrupt either the BPL signal or the other signals.

A detailed analysis of such phenomena is carried out here, taking into account the characteristics of all the signals carried by this cable during regular train operation, with the scope of assessing the real feasibility of such application, ensuring that both new signals (used for example for infotainment) and standard signals carried by the cable do not interact with each other.

It is fundamental to underline that these result show eventual theoretical feasibility of the PLC implementation, but a thorough experimental measurement campaign is needed to assess the simulation results and verify the practical feasibility.

\section{System description}

In this section the main physical characteristics of the UIC cables are given, together with an analysis of the functions they are supposed to operate. The electrical parameters of the cable are obtained both by simulations and measurements and a model of the whole systems is proposed.

\subsection{UIC cables description}

As described in [8] most trains running on European tracks are equipped with UIC cables, being the main core of the remote control and communication line. In particular an 18 conductors flexible shielded cable (terminated by a plug) connects the stocks one another and it is connected (inside each stock) to a connection box (present at both ends of each vehicle). Inside the vehicle the 18 conductors are divided in two different cables: a 16 conductors and a two conductor cables, both shielded. Figure 1 shows the section of the 16 conductors cable with the cable numbering as explained in [8]. 


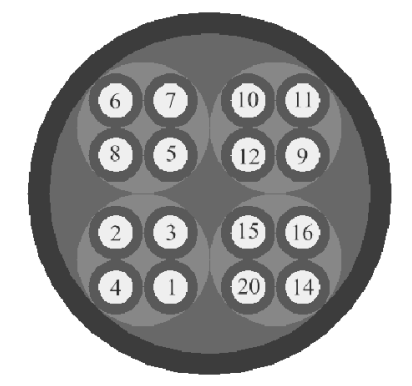

Figure 1: Section of the 16 conductors cable

The 16 conductors have different roles in the train control, and they are divided in functional groups: each single group has one conductor serving as a reference and one or more conductors as signal line. A brief description of the role of the conductors is the following:

- Conductors 1 to 8 are by default dedicated to the transmission of voice signals and are characterized by AC signals at a frequency range $[100,8000] \mathrm{Hz}$ and permanent DC signals characterized by a value between 18 and $33 \mathrm{~V}$.

- Conductors 9 to 16 are by default dedicated to the transmission of DC pulses (with amplitude between 15 and $33 \mathrm{~V}$ and 2 seconds duration) for doors opening and closing and for the light signals "stop" and "go", with conductor \#12 as common ground.

In addition, some users (DB, ÖBB, CFF, SNCF) utilize conductors 9 to 12 for additional functions: in particular

- Conductors 9 to 12 are dedicated to the emergency brake and the electropneumatic actuators (DC pulses with value between 15 and $33 \mathrm{~V}, \mathrm{DB}$, ÖBB, CFF).

- Conductors 9 and 10 can carry an $\mathrm{AC}$ signal at $1 \mathrm{kHZ}$ relative to the emergency brake information (DB, ÖBB, CFF).

- Conductors 10 and 11 carry AC signals (around $100 \mathrm{kHz}, 600-950 \mathrm{mV}$ ) relative to traction commands (DB).

- Conductors 7, 9,10 and 11 carry additional signals in case the train has to leave the station in the opposite direction of its arrival $(6 \mathrm{~V}$, frequency band $[10-20$ $\mathrm{kHz}]$ (SNCF).

- Conductors 7 and 8 are used for voice message in case there are external loudspeakers (DC signal, between 18 and 33V, SNCF).

All the above conductors are good candidates to carry the broadband powerline channel, since standard OFDM protocols (such the widely diffused HomePlug AV) work in the frequency range [2 - 30] Mhz and there is no overlapping in the frequency bands above mentioned.

In [9] and [10] the authors chose conductor $\# 12$ as a return and one of the conductors \#10 or \#11 (dedicated to the switching on and off of the light signals "stop" and "go") because of the specific default application ("stop" and "go" signal) with the application to Italian national railway system.

Taking into account also the additional functions above described, the previous choice could still be used, but in this paper we investigate a different choice: the 
selected two conductors line is composed by conductors 5 and 6 . This couple of conductors is dedicated to the switching of the speakers and is characterized by DC signals of high magnitude $(15-33 \mathrm{~V})$ which are not affected by the low power OFDM signal in a high frequency band.

\subsection{UIC cable modelling and measurements}

The first step to be performed when modeling a multiconductor transmission line is to obtain its per unit length parameters. It is worth mentioning at this point that, due to the OFDM frequency range, it is of fundamental importance, in order to correctly evaluate the channel's performance, to characterize the frequency dependence of the parameters. For this reason an accurate 2D FEM model of the cable section (Figure 1) has been implemented, obtaining the $R, L$ and $C$ matrices. In this case the frequency range typical of the OFDM protocol is between 2 and $30 \mathrm{MHz}$, for this reason the p.u.l. parameters have been calculated for 3 frequencies in the above mentioned range, and the frequency behavior has been modeled as described in [11]. In order to validate the simulations a measurement campaign has been carried out on a $6 \mathrm{~m}$ UIC cable provided by Trenitalia S.p.a. In particular the measurements have been performed on a couple of conductors whose distance was minimum (i.e. 9-11 or 14-16). The p.u.l. behavior in a range [0 30] $\mathrm{MHz}$ is shown in figures $2-5$, showing good agreement with the parameters obtained by the FEM simulation.

The relative error between measurements and simulations in the frequency range of interest is below $10 \%$. Some small divergences are caused by the measurement system, but the global frequency behavior of the measured p.u.l. parameters is well reproduced by the FEM model.

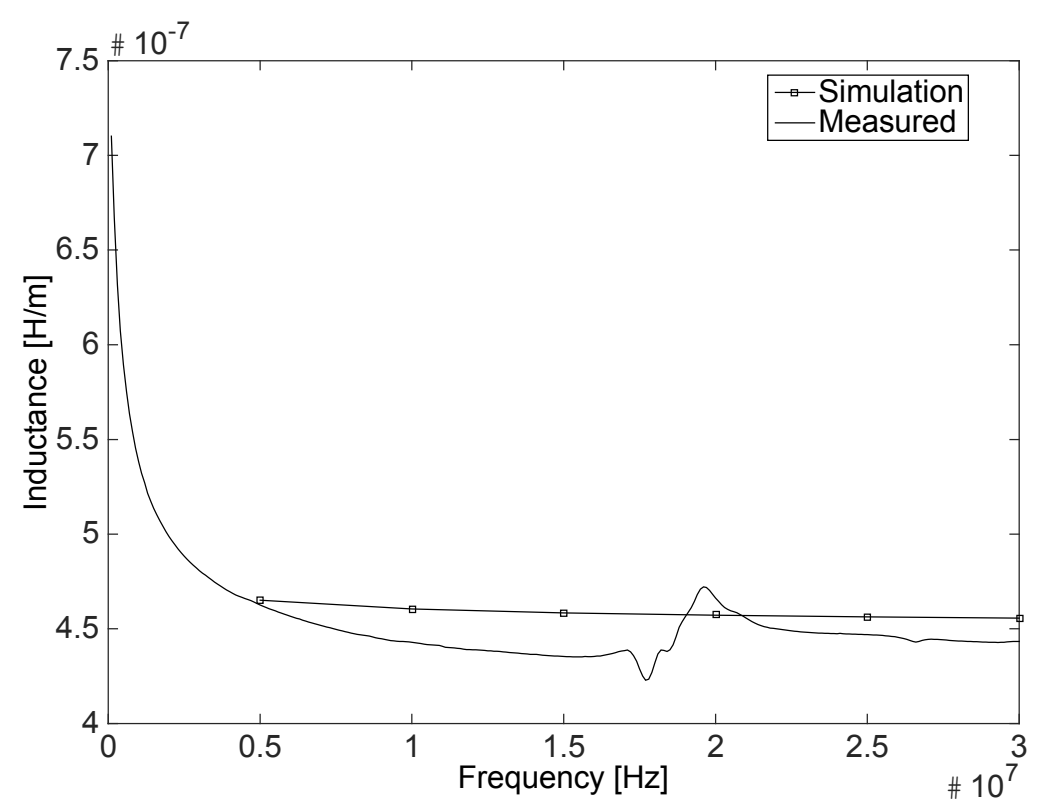

Figure 2: Measured and simulated inductance 
While the p.u.l. L, R and C have been both calculated and measured, the simple FEM model could not allow us to calculate the conductance (mainly because the dispersive properties of the insulating material composing the cable were not known); for this reason, the value of $\mathrm{G}$ used in the channel simulations is taken directly from the measurements.

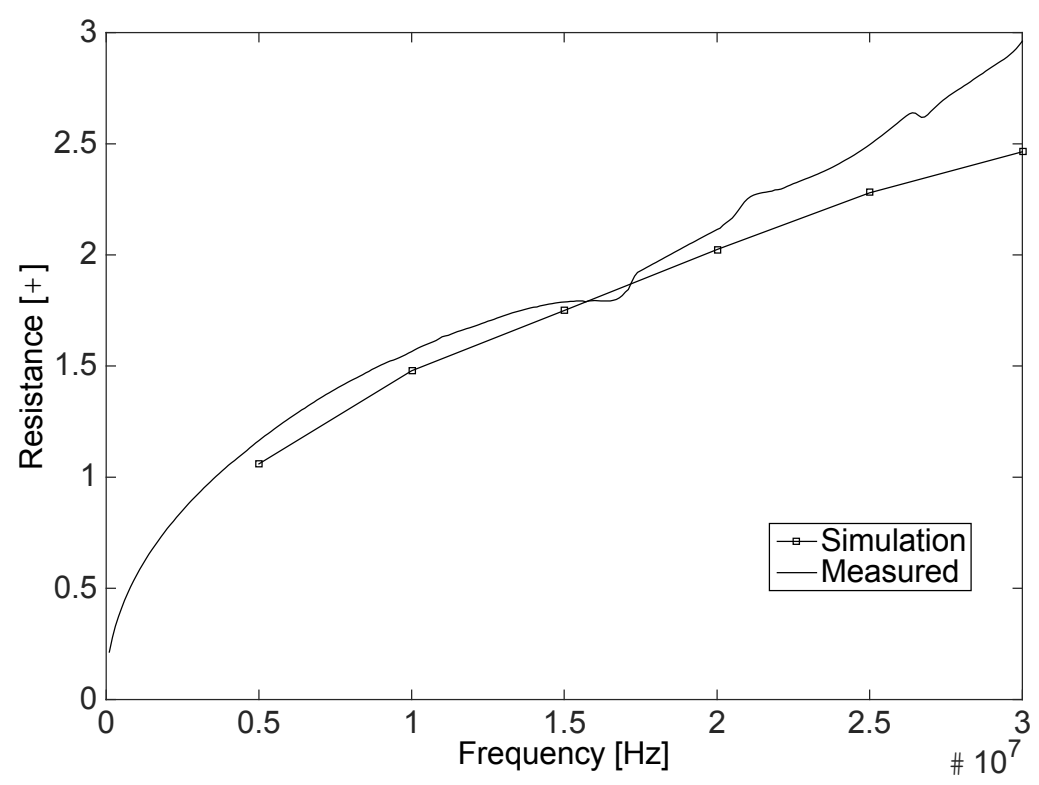

Figure 3: Measured and simulated resistance

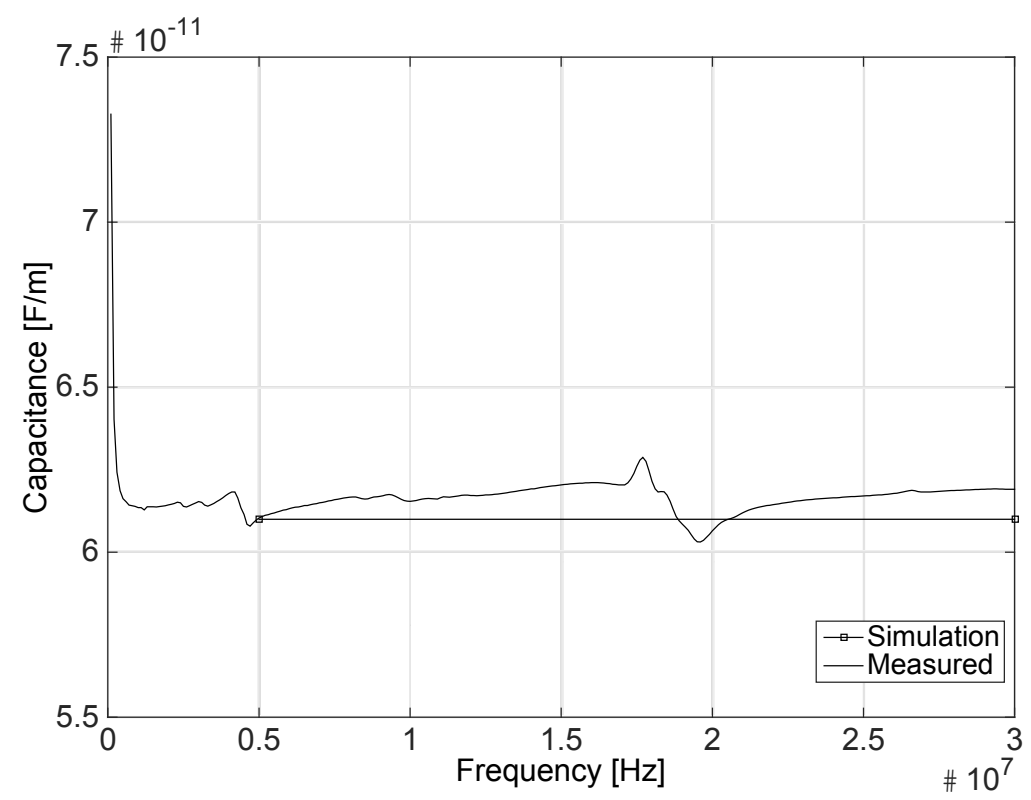

Figure 4: Measured capacitance 


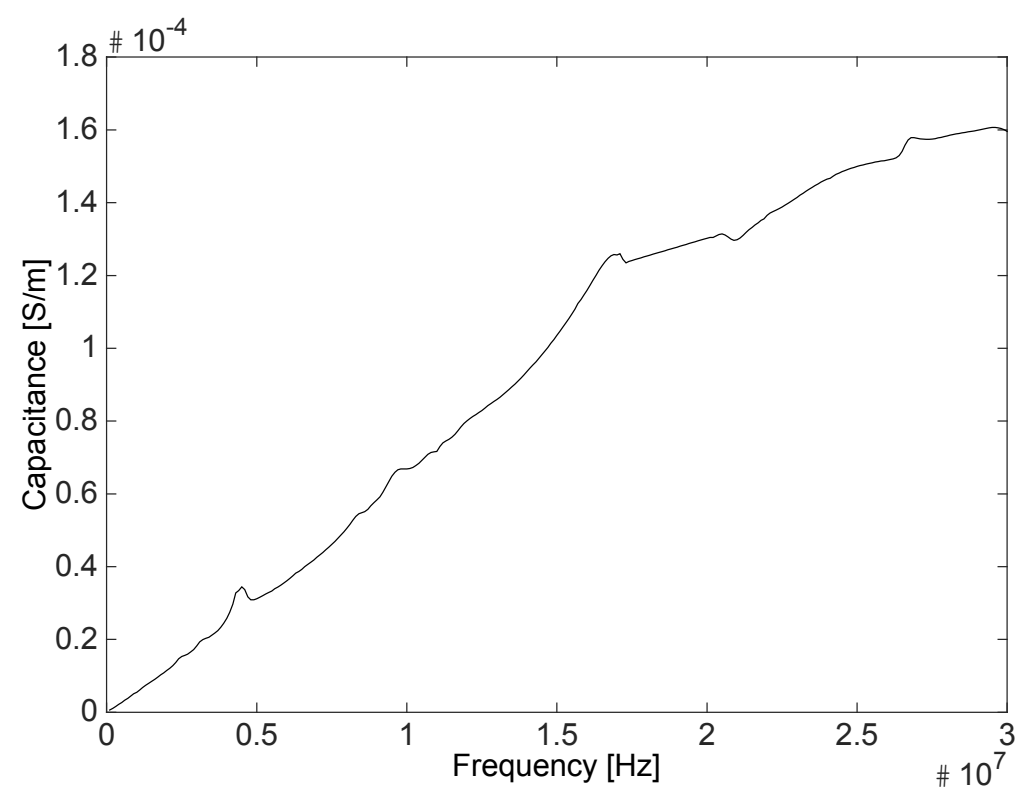

Figure 5: Measured conductance

\subsection{Simulated System}

The whole system is a Multiconductor Transmission Line, whose length is given by the number of hauled stock composing the train. The electrical length of the cable inside each stock is about $1=20 \mathrm{~m}$; furthermore, additional $5 \mathrm{~m}$ can be considered the length of the connection between the two coaches. A model of 7 hauled stocks has been considered $($ length $=175 \mathrm{~m})($ see Figure 6$)$.

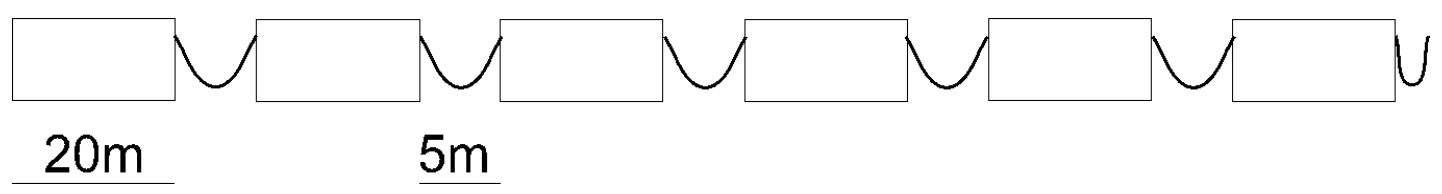

Figure 6: Outline of the simulation

At the moment, the cable is terminated with an open circuit, but in order to optimize the communication performances a different termination (typically the characteristic impedance).

Each single cable is dedicated to different functions; it is hence characterized by a termination impedance. Their value is not described in [8], with the exception of conductors \#9 to \#15 in which a shunt resistance higher than $1200 \Omega$ is noted.

Each hauled stock should be equipped with a PLC modem capable of receiving/sending the PLC signal; these modems typically have a $50 \Omega$ resistance, for this reason in this paper a $50 \Omega$ shunt resistance has been considered in each hauled stock. Any resistance of higher value, such as the ones mentioned before, have a negligible effect on the data transmission.

In the next section the results of the simulations are shown. 


\section{Simulations results}

The simulations are here performed in the frequency domain because our main goal is to obtain the transfer function (between transmitter and receiver) in order to evaluate signal attenuation and crosstalk in the whole frequency range typical of PLC applications.

\subsection{Frequency Response and Crosstalk}

Figure 7 shows the transfer function magnitude between the $n^{\text {th }}$ (with $n=1$ to 7 ) hauled stock and the first one (where the transmitter modem is supposed to be placed) in the frequency range [2-30] $\mathrm{MHz}$.

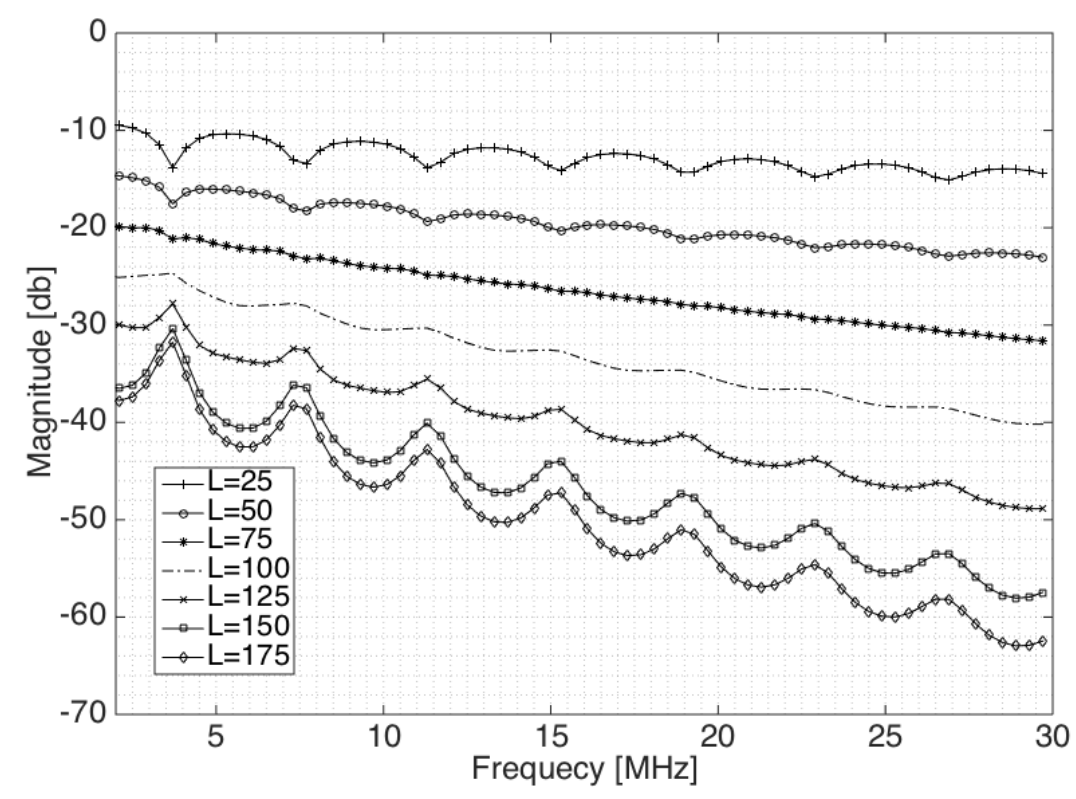

Figure 7: Transfer functions between different hauled stocks

It can be observed that, as it is easy to predict, attenuation increases with distance from the modem and with frequency. Additional information which can be drawn from Figure 7 is the following: considering the maximum practicable attenuation of about $-30 \mathrm{db}$, a repeater is needed every three hauled stocks if the whole frequency band is needed. 


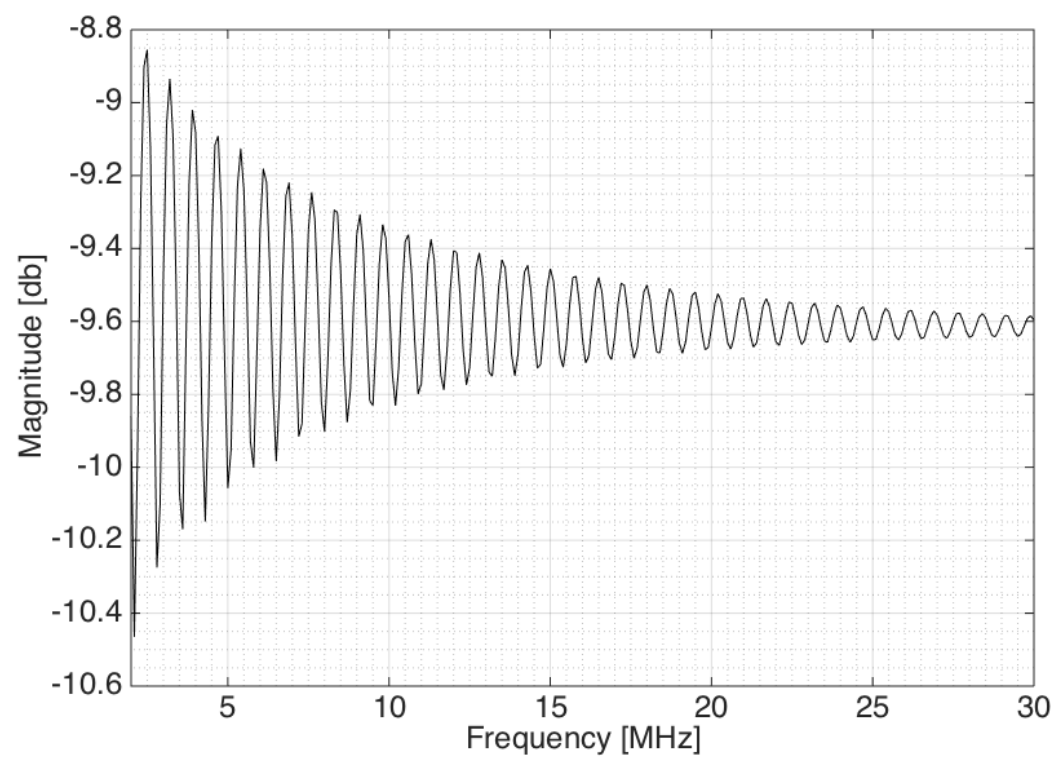

Figure 8: Near end crosstalk (open circuit)

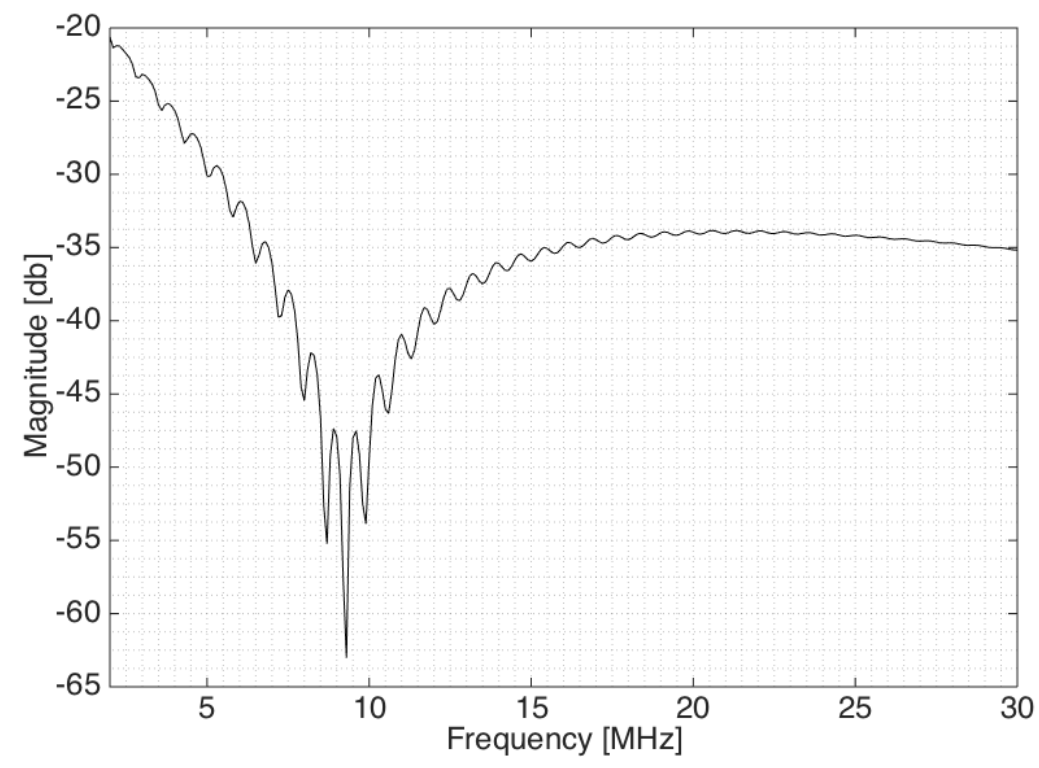

Figure 9: Far end crosstalk (open circuit)

Figure 8 and 9 show the near end and far end crosstalk relative to conductor 7 (with 6 as common ground) when conductor 5 is excited and the terminations of conductor 7 being set as open circuit. Considering the magnitudes shown in the above figures it is evident that at far end the presence of the PLC signal is highly attenuated (more than $30 \mathrm{db}$ in most frequency band) and at near end the average attenuation of $9.6 \mathrm{db}$ is still satisfactory taking into account the magnitudes of the signals described in section 2.1. 


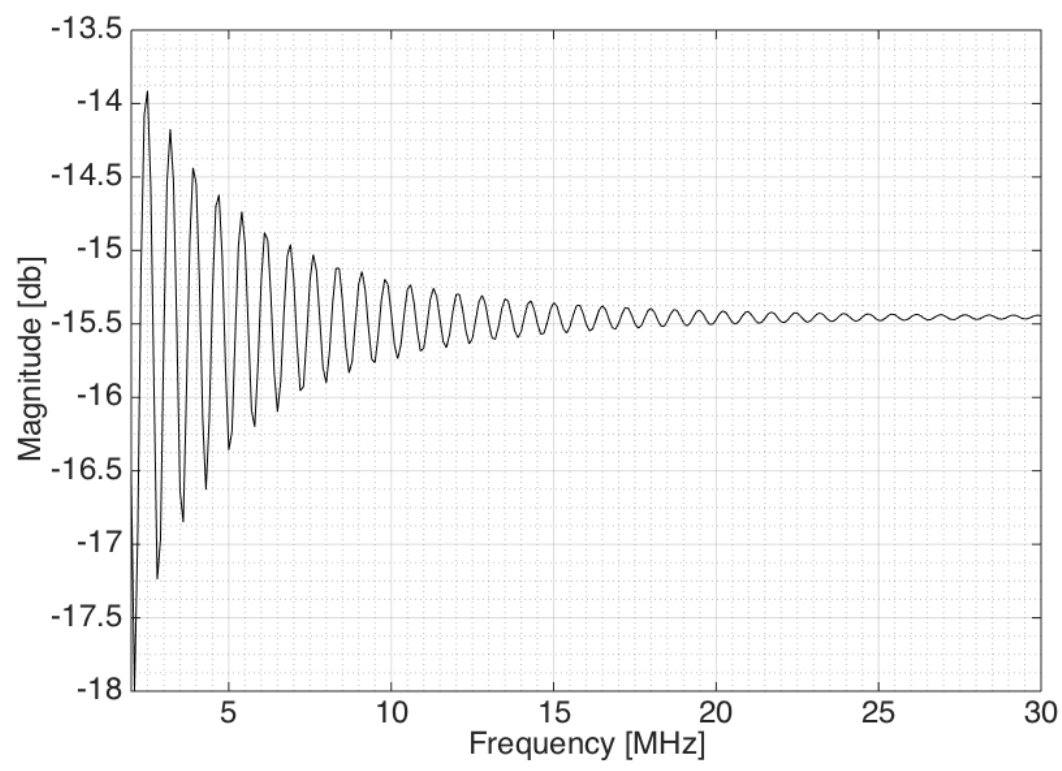

Figure 10: Near end crosstalk (characteristic impedance)

The open circuit situation analyzed above is in most cases the worst in terms of crosstalk magnitude. Figures 10 and 11 show the transfer function relative to the same conductors but with the terminations of conductor 7 set to the characteristic impendence (around 86 2 ): in this case both near and far end attenuations are higher (due to the lack of reflections at the termination) resulting in an even more reduced effect of the PLC signal on lines carrying other signals.

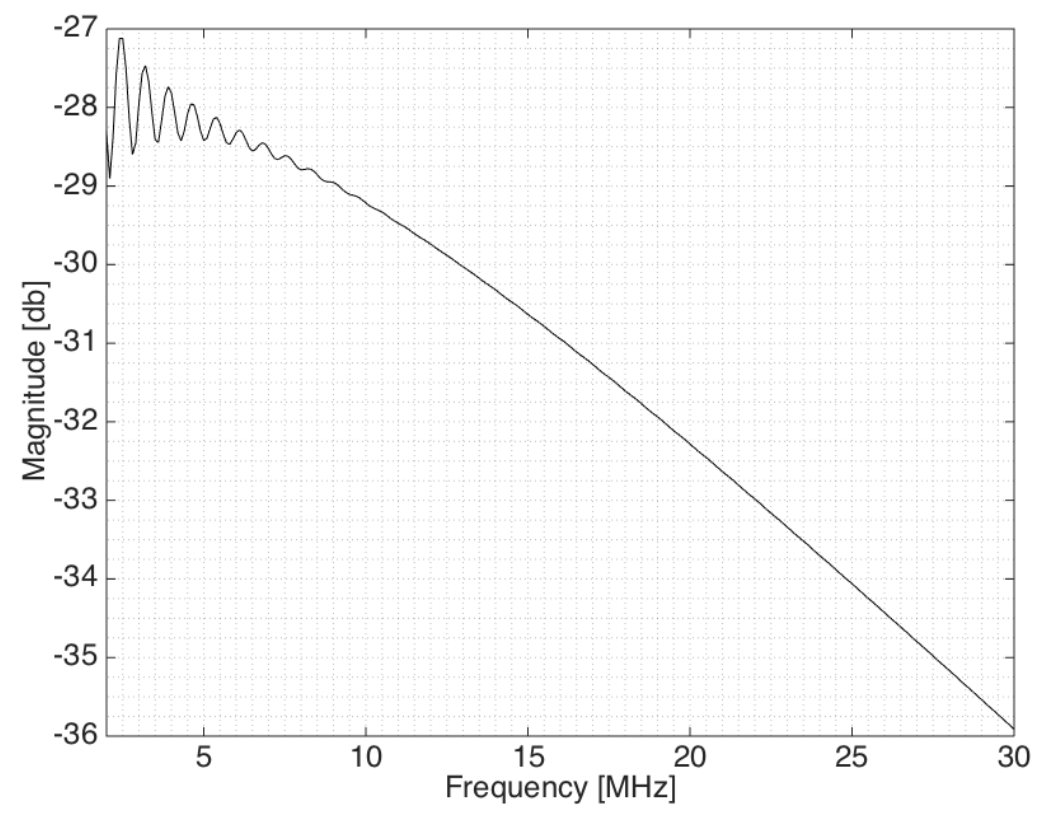

Figure 11: Near end crosstalk (characteristic impedance) 


\subsection{Channel Capacity}

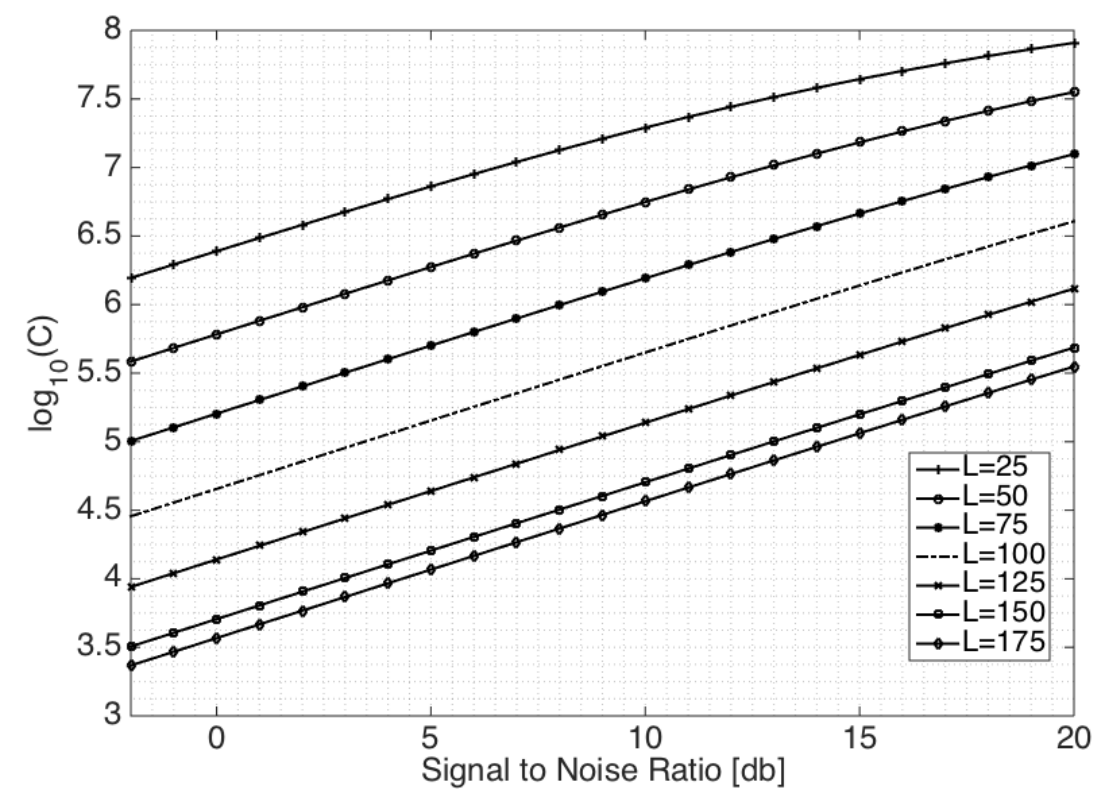

Figure 12: Theoretical channels capacities as a function of the signal to noise power ratio.

By using the Shannon-Hartley theorem, we calculated the theoretical capacities of the seven channels whose frequency responses are shown in Fig.7. The channel capacity is expressed as a function of the signal to noise power ratio $S / N$, where $S$ and $N$ represent, respectively, the power of the transmitted signal and the power of an additive white Gaussian noise at the receiver. We consider both $S$ and $N$ independent from frequency, then then the power of the received signal is given by $|H(f)|^{2} S$, where $H(f)$ denotes the frequency response of the channel, and the capacity of the channel may be written as:

$$
C\left(\frac{S}{N}\right)=\int_{B_{\min }}^{B_{\max }} \log _{2}\left(1+|H(f)|^{2} \frac{S}{N}\right) d f
$$

where the capacity is expressed in bits per second.

A numerical calculation of (1) was performed using a trapezoidal interpolation, and considering $B_{\min }=2 \mathrm{MHz}$ and $B_{\max }=30 \mathrm{MHz}$, i.e. the bandwidth limits of the Homeplug AV protocol that uses OFDM. We note that the performance of the OFDM modulation method has been reported to be, in general, very close to the limit given by the theoretical channel capacity as calculated in (1).

Figure 12 shows the resulting channel capacities for increasing distance from the transmitter, considering signal to noise power ratio (SNR) values from -2 to $20 \mathrm{db}$. 
Considering a highly noisy scenario of $\mathrm{SNR}=0 \mathrm{db}$, the capacity of the shortest path channel $(25 \mathrm{~m})$ is circa $2.5 \mathrm{Mbit} / \mathrm{s}$, and the capacity of the longer path channel $(175 \mathrm{~m})$ is $3.7 \mathrm{kbit} / \mathrm{s}$. In a low noise scenario $(\mathrm{SNR}=20 \mathrm{db})$ the capacities increase up to $35 \mathrm{kbit} / \mathrm{s}$ for the long path and $80 \mathrm{Mbit} / \mathrm{s}$ for the long path. Moving from one coach to the successive one the gain loss is in average $6 \mathrm{db}$, and from figure $12 \mathrm{we}$ have a confirmation that for distances longer than $75 \mathrm{~m}$ a repeater is necessary to maintain an acceptable quality of service. In addiction for shorter distances the channel is adequate for a high speed broadband transmission, for example for video broadcasting and infotainment applications.

\section{Conclusions}

In this paper the authors investigate the use of UIC cables, already present on board most trains, to send high speed data to be used as additional services for passengers or security/safety set ups.

The analysis is carried out taking into account all fundamental functions of the UIC cables in order to guarantee that the relative signals are not disrupted by the additional data transfer required to implement a broadband power line transmission. The results show that the signal integrity of pre-existing data is kept and that a high speed connection is achievable.

These results are relative to a feasibility study and are anyway subject to test field in order to verify practical realization.

\section{References}

[1] P. A. J. van Rensburg, H. C. Ferreira,A. J. Snyders, "An experimental setup for in-circuit optimization of broadband automotive power-line communications", ISPLC 2005, 6-8 April 2005, pp. 322 - 325.

[2] T. Huck, J. Schirmer, T. Hogenm, K. Dostert, "Tutorial about the implementation of a vehicular high speed communication system" ISPLC 2005, 6-8 April 2005, pp. 162 - 166.

[3] C. H. Jones, "Communication over aircraft power lines" ISPLC 2006, 26 - 29 March 2006, pp. 149 - 154.

[4] V. Degardin, P. Laly, M. Lienard, P. Degauque, "Impulsive noise on invehicle power lines: characterization and impact on communication performance", ISPLC 2006, 26 - 29 March 2006, pp. 222 - 226.

[5] V. Degardin, P. Laly, M. Lienard, P. Degauque, "Performances of the HomePlug PHY Layer in the Context of in-vehicle Powerline Communications", ISPLC 2007, 26 -28 March 2007, Pisa, Italy pp. 93 - 98.

[6] E. Liu, Y. Gao, G. Samdani, O. Mukhtar, T. Korhonen, "Powerline Communication over Special Systems", ISPLC 2005, 6 -8 April 2005, Vancouver, Canada pp. 167 - 171.

[7] S. Tsuzuki et. al. "Characteristics of Power-Line Channels in Cargo Ships", Proceedings of ISPLC 2007, 26 -28 March 2007, Pisa, Italy pp. 324 - 32. 
[8] UIC 558, "Ligne de telecommande et d'information. Caracteristiquest techniques unifiees por l'equipment des voitures RIC", Union Internationale des Chemins de fer.

[9] S. Barmada, A. Gaggelli, P. Masini, A. Musolino, R. Rizzo and M. Tucci "Modeling of UIC Cables in railway Systems for their Use as Power Line Communications Channels", Applied Computational Electromagnetics Journal, vol. 24, no. 6, December 2009.

[10] S. Barmada, A. Gaggelli, A. Musolino, R. Rizzo, M. Raugi and M. Tucci "Design of a PLC System Onboard Trains: Selection and Analysis of the PLC Channel”, Proceedings of ISPLC 2008, 2 -4 April 2008, Jeju Island, Korea.

[11] C. R. Paul "Analysis of Multiconductor Transmission Lines", Wiley, 1994. 